\section{Bewertung des oronasalen Flusses mittels Temperatur (Thermistor) und Staudruck (Prongs)}

K.-H. Rühle, A. Fahrner, W. Randerath

Klinik für Pneumologie, Klinik Ambrock, Universität Witten/ Herdecke, Hagen
Zusammenfassung: Die Messung des oronasalen Flusses mit Thermistoren gilt im Rahmen einer Polysomnographie als Standard und ist international weit verbreitet. Alternativ besteht die Möglichkeit, den während der Atmung in einer Nasenbrille (Prongs) entstehenden Staudruck zu messen. Ziel dieser Arbeit soll es sein, die heute in der klinischen Routine möglichen Methoden, nämlich

- $\mathrm{O}_{2}$-Sättigung mittels Pulsoxymetrie

- oronasaler Fluss mittels Thermistor

- thorako-abdomineller Effort mittels Gurten

- oronasaler Fluss mittels Doppelprongs, zu vergleichen und einzustufen.

8 konsekutive Patienten mit Verdacht auf Schlafapnoesyndrom. (7 m, $1 \mathrm{w}$ ), mittleres Alter 56,5 \pm 9,5 Jahre, Größe $173 \pm 6,4 \mathrm{~cm}$, BMI 28,7 $\pm 2,6 \mathrm{~kg} / \mathrm{m}^{2}$, Score der Epworth-Sleepiness-Scale 9,1 \pm 3,2 , AHI $24,9 \pm 13,7 \mathrm{~h}^{-1}$, tiefste $\mathrm{SaO}_{2} 86 \pm 4,9 \%$, wurden untersucht. Neben einem oronasalen Dreipunkt-Thermistor wurde ein oronasaler Brillenschlauch (zwei Öffnungen für die Nase, zwei Öffnungen für den Mund, ein gemeinsamer Druckschlauch) verwandt. Der entstehende Staudruck wurde über einen Druckwandler parallel zum Thermistorsignal und den anderen polysomnographischen Daten registriert.

Als respiratorisches Ereignis wurde dieses gezählt, wenn zumindest ein Kriterium einer respiratorischen Störung (exakte Definition siehe Methodik) erfüllt wurde. Alle derart definierten respiratorischen Ereignisse wurden zu einer Grundgesamtheit addiert. Die Detektionsraten der vier Messmethoden wurden bezogen auf die Grundgesamtheit der Ereignisse ermittelt.

Von den 1824 respiratorischen Ereignissen (100\%) wurden lediglich 52,3\% aller Ereignisse mittels einer $\mathrm{O}_{2}$-Desaturation von wenigstens $4 \%$ entdeckt, dagegen $61,0 \%$ mittels ThermistorSignal. Die zweithöchste Detektionsrate fand sich bei der Messung des Effort mit $83,4 \%$, die höchste Detektionrate ergab die Registrierung mittels Prongs mit 95,1\%.

Zusammenfassend hat sich die Staudruckmessung der Atmung in unseren Untersuchungen gegenüber der Thermistor-Methode als deutlich überlegen gezeigt.

Evaluation of Oronasal Flow by Means of Temperature (Thermistors) and Nasal Cannula Pressure (Prongs): The measurement of oronasal flow during sleep studies using thermistors is internationally well accepted. As an alternative the

Pneumologie 2001; 55: 4-6

(c) Georg Thieme Verlag Stuttgart · New York ISSN 0934-8387 possibility exists to measure the pressure at the nose by means of nasal prongs.

Our objective was to compare and evaluate the methods to detect respiratory events in clinical routine, namely

- $\mathrm{O}_{2}$-saturation by pulse oxymetry

- oronasal flow by thermistors

- thoraco-abdominal effort by belts

- oronasal flow by prongs,

8 consecutive patients suspected of sleep apnoea syndrome $(7 \mathrm{~m}, 1 \mathrm{f})$, mean age $56.5 \pm 9.5$ years, height $173 \pm 6.4 \mathrm{~cm}$, BMI $28.7 \pm 2.6 \mathrm{~kg} / \mathrm{m}^{2}$, Epworth sleepiness scale score $9.1 \pm 3.2$, AHI $24.9 \pm 13.7 \mathrm{~h}^{-1}$, lowest $\mathrm{SaO}_{2} 86 \pm 4.9 \%$, were examined.

Between nose and mouth we fixed an oronasal 3-point thermistor and oronasal prongs ( 2 openings at the mouth and 2 openings at the mouth applying a common tube). During breathing the resulting pressure was registered with a pressure transducer parallel to the signal of the thermistors and the other polysomnographic data. We defined a respiratory event if at least one of the 4 signals met the criteria of a respiratory disturbance (for exact definitions see method).

The detection rate of the 4 signals was calculated in relation to the sum of all events.

Of the 1824 events ( $100 \%$ ) only $52.3 \%$ were detected by an $\mathrm{O}_{2}$ desaturation of more than $4 \%$, but $61.0 \%$ by thermistors. An acceptable detection rate was found measuring effort with $83.4 \%$, only nasal prongs detected $95.1 \%$ of all events.

We conclude that with regard to the detection rate of respiratory events the measurement of pressure by nasal prongs is superior to the use of thermistors.

\section{Einleitung und Fragestellung}

Der oronasale Fluss wird zur Zeit in der klinischen Routine im Rahmen einer Polysomnographie üblicherweise mit Thermistoren registriert. Diese Messmethode gilt als Standard und ist international weit verbreitet. Allerdings wurden in den letzten Jahren erhebliche Zweifel an der Bewertbarkeit der Messdaten dieser Sensoren geäußert. Mögliche Störfaktoren sind unterschiedliche Atemfrequenz, unterschiedlicher Kurvenverlauf des Atemflusses und Abstand des Thermistors von Mund und Nase, wodurch die Flussmessung verfälscht wird. Außerdem wurde die Tatsache kritisiert, dass bedingt durch die alineare Beziehung zwischen mit dem Thermistor und dem pneumotachographisch (Referenz) gemessenen Fluss erhebliche Fehler im Hinblick auf die Bewertung von Apnoen und Hypopnoen in der Diagnostik des obstruktiven SchlafapnoeSyndrom (OSAS) auftreten können [6]. 
Die Alternative, den oronasalen Fluss mit dem Pneumotachographen zu messen, hat den Vorteil, dass sie allgemein als Goldstandard in der Flussmessung akzeptiert wird [8].

Allerdings führt die hierfür erforderliche Maske zu erheblichen Problemen bei der Akzeptanz.

Eine weitere Möglichkeit, den oronasalen Fluss zu messen, besteht darin, den während der Atmung in einer Nasenbrille (Prongs) entstehenden Staudruck zu messen [9]. In einer klinischen Studie konnten Ballester u. Mitarb. (1998) [3] zeigen, dass der Einsatz von Prongs die Detektion respiratorischer Ereignisse gegenüber Thermistor und thorakalen bzw. abdominellen Gurten deutlich verbessern konnte.

Ziel dieser Arbeit soll es sein, die heute in der klinischen Routine möglichen Methoden, nämlich

- $\mathrm{O}_{2}$-Sättigung mittels Pulsoxymetrie

- oronasaler Fluss mittels Thermistor

- thorako-abdomineller Effort mittels Gurten

- oronasaler Fluss mittels Doppelprongs,

zu vergleichen und einzustufen.

\section{Methodik}

\section{Patienten}

Ausgewählt wurden 8 konsekutive Patienten ( $7 \mathrm{~m}, 1 \mathrm{w})$, mittleres Alter 56,5 \pm 9,5 Jahre, Größe $173 \pm 6,4 \mathrm{~cm}$, BMI 28,7 $\pm 2,6 \mathrm{~kg} / \mathrm{m}^{2}$, Score der Epworth-Sleepiness-Scale 9,1 $\pm 3,2$, AHI $24,9 \pm 13,7 \mathrm{~h}^{-1}$, tiefste $\mathrm{SaO}_{2} 86 \pm 4,9 \%$, die sich mit Verdacht auf Schlafapnoesyndrom in unserer Klinik vorgestellt hatten.

\section{Design}

Alle Patienten wurden im Schlaflabor polysomnographisch nach DGSM-Kriterien abgeleitet und das Hypnogramm nach Rechtschaffen und Kales ausgewertet. Das Ergebnis der Schlafstadienanalyse kann Tab.1 entnommen werden. Neben einem oronasalen Dreipunkt-Thermistor (Fa. Delta Regeltechnik, München, Modell 5K3A1I) wurde ein oronasaler Brillenschlauch (zwei Öffnungen für die Nase, zwei Öffnungen für den Mund, ein gemeinsamer Druckschlauch, Fa. Salter-Labs, Arvin, CA/USA, Modell REF 4001) verwandt (siehe Abb.1). Der entstehende Staudruck wurde über einen Druckwandler (Fa. Compumedics/ResMed, Mönchengladbach) parallel zum Thermistorsignal und den anderen polysomnographischen Daten registriert.

\section{Auswertung}

Apnoe wurde definiert als Verminderung des Flows unter 10\% des Ausgangsignals (Thermistor und/oder Prongs) für länger als 10 Sekunden. Hypopnoe wurde definiert als Verminderung des Ausgangsignals um mehr als 50\% (Thermistor und/oder Prongs). Ein Ereignis wurde weiterhin dann gewertet, wenn eine $>50 \%$ ige Reduktion eines Effortkanals und oder eine $>50 \%$ ige Reduktion des Thermistor-Signals und/oder $>50 \%$ ige Reduktion des Prongs-Signals oder Reduktion der Sauerstoffsättigung um wenigstens $4 \%$ auftrat. Die Detektionsraten der

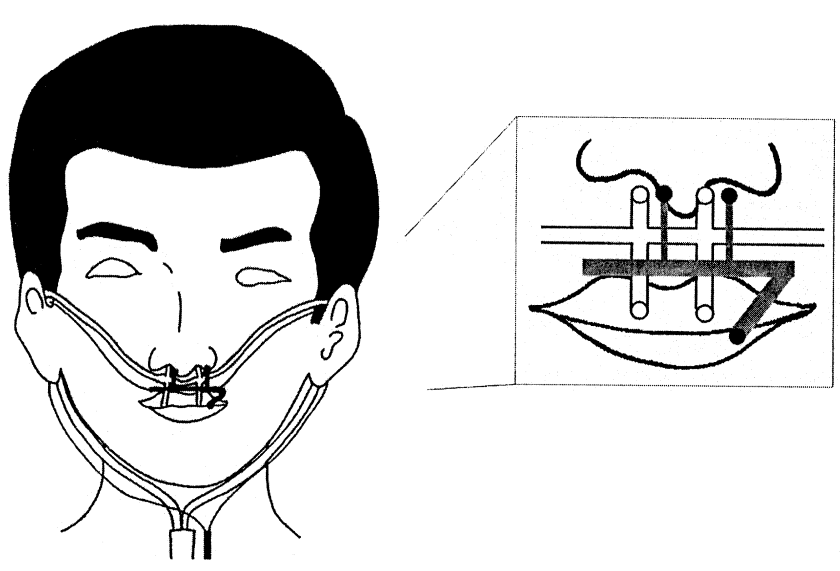

Abb. 1 Schema der oronasalen Flussmessung. Das Signal des Dreipunkt-Thermistors und der oronasalen Doppelprongs werden gleichzeitig gemessen.

vier Messmethoden wurden bezogen auf die Grundgesamtheit der Ereignisse ermittelt.

\section{Ergebnisse}

Die Daten der Schlafstadienanalyse sind Tab. 1 zu entnehmen.

Tab. 1 Ergebnis der Schlafstadienanalyse

\begin{tabular}{lllllllll}
\hline & TIB & TST & S1 & S2 & S3/4 & REM & WASO & Effizienz \% \\
\hline MW & 402 & 321 & 48,8 & 185 & 35,9 & 50,9 & 46,7 & 79,7 \\
SD (min) & 30,4 & 34,6 & 28,0 & 40,2 & 41,0 & 17,4 & 19,0 & 5,9 \\
\% TST & & & $15 \%$ & $58 \%$ & $11 \%$ & $16 \%$ & & \\
\hline
\end{tabular}

TIB = Time in Bed; TST = Gesamtschlafzeit; WASO = Wachzeit nach Schlafbeginn

Von den 1824 Ereignissen (100\%) wurden lediglich 52,3\% aller Ereignisse mittels einer Sauerstoffsättigungsminderung von wenigstens $4 \%$ entdeckt, dagegen $61,0 \%$ mittels ThermistorSignal (s. Tab. 2). Die zweithöchste Detektionsrate fand sich bei der Messung des Effort mit 83,4\%, die höchste Detektionsrate ergab die Registrierung mittels Prongs mit 95,1\%.

Tab. 2 Die Detektionsraten der Messmethoden bezogen auf die Grundgesamtheit

\begin{tabular}{lll}
\hline & \% aller Ereignisse & AHI \\
\hline $\mathrm{SaO}_{2}$ & 52,3 & $21,2 \pm 13,7$ \\
Thermistor & 61,0 & $24,9 \pm 13,7$ \\
Effort & 83,4 & $34,3 \pm 15,2$ \\
Prongs & 95,1 & $39,0 \pm 16,0$ \\
\hline
\end{tabular}




\section{Diskussion}

Um möglichst viele Ereignisse zu erfassen, genügte für die Wertung als Apnoe/Hypopnoe, wenn schon allein ein Kriterium erfüllt wurde. In der Auswertung wurden diese Ereignisse zu einer Grundgesamtheit zusammengefasst. Ähnlich wie Ballester u. Mitarb. [3] fanden wir ohne Berücksichtigung von Arousals die höchste Detektionsrate unter einer Messung mit Prongs. Während die genannte Arbeitsgruppe eine Detektionsrate von $96,8 \%$ ermittelte, lag diese in unserer Studie bei $97,1 \%$ und damit geringfügig höher.

Während die Kriterien für Apnoe und Hypopnoe mit Prongs von Ballester u. Mitarb. nur qualitativ beurteilt wurden („change of pattern“), werteten wir die respiratorischen Ereignisse nach quantitativ beschreibbaren Kriterien. Sie bewerteten lediglich zwei Stunden einer Nachtmessung, wobei die erste und die letzte Stunde ausgeschlossen wurden. Unsere Untersuchungen beziehen sich auf die komplette Nachtmessung, um Allgemeingültigkeit für die klinische Routine zu erhalten.

In einer Studie von Gleeson u. Mitarb. [7] konnte nachgewiesen werden, dass bei Männern in $29 \pm 8,2 \%$ der gesamten Schlafzeit durch den Mund geatmet wurde. Da Ballester et al. nur nasale Prongs verwandten, könnten falsch positive respiratorische Ereignisse durch die ausschließliche Erfassung des nasalen Flusses resultieren. Da wir dagegen oronasale Doppelprongs einsetzten und bei vermindertem nasalen Fluss und konsekutiver Mundatmung das oronasale Summensignal normalen Fluss anzeigt, kann bei dieser Methodik ausgeschlossen werden, dass fälschlicherweise Hypopnoen registriert werden.

Aufgrund der höheren Detektionrate insbesondere im Vergleich zu Thermistoren könnte die Staudruckmethode bei grenzwertigen Befunden oder upper-airway-resistance-syndrome $[5,10]$ eingesetzt werden.

Eine vergleichende Untersuchung aus der Arbeitsgruppe Rappoport [2] weist darauf hin, dass die Prongs-Methode eine gleich gute Entdeckungsrate von mittels Ösophagusdruckmessungen definierten respiratorischen Arousals besitzt. Dies ist unter anderem der Grund dafür, dass die Taskforce der American Association of Sleep Medicine [1] empfiehlt, dass die Druckmessung mit Prongs als alleinige zuverlässige Methode zur Flussmessung benutzt werden soll.

Die praktische Bedeutung der Prongs-Methode zeigt sich beispielhaft darin, dass bei einem Patienten mittels Thermistor ein AHI von 8,7, mittels Prongs dagegen ein AHI von 20,6 ermittelt wurde. Unter alleiniger Berücksichtigung des Thermistors wären in diesem Fall erhebliche Zweifel an der Ursache der Symptomatik des Patienten und damit an der CPAP-Indikation anzumelden gewesen, zumindest wenn ein AHI-Grenzwert von 10 pro Stunde angesetzt wird.

Einschränkend sollte festgehalten werden, dass bei RoutineUntersuchungen das Prongs-Signal nur zeitweise verwertbar ist. In einer unserer weiteren Studien (unveröffentlichte Ergebnisse) lag die Ausfallrate bei der Staudruckmethode bei $33 \%$ aller Messungen. Dies dürfte unter anderem auf eine fehlerhafte Position der Prongs als auch auf die mögliche
Verstopfung der Öffnungen durch Sekret bedingt gewesen sein. Die Daten des Thermistors dagegen waren in allen Fällen auswertbar. Damit wird der Vorteil der hohen Detektionsrate mittels Prongs durch eine geringere Zuverlässigkeit im Vergleich zur Thermistor-Methode vermindert.

Als relativ sensitiver Detektions-Parameter bei OSAS erwies sich die Veränderung der Effort-Kanäle [4].

Mit dem erwähnten Kriterium einer 50\%-Reduktion des Effort lag die Erkennungsrate deutlich über derjenigen des Thermistorsignals. Dieser Parameter besitzt deshalb bei guter Registrierqualität im Vergleich zum Thermistor eine hohe Bedeutung in der Beurteilung von SBAS.

Zusammenfassend hat sich die Staudruckmessung der Thermistor-Methode als deutlich überlegen gezeigt. Um die im Schlaf nicht selten auftretende Mundatmung zu erfassen und grenzwertige Befunde besser einordnen zu können, empfiehlt es sich, oronasale Doppelprongs zu verwenden. Die Thermistor-Methode erweist sich aufgrund ihrer Zuverlässigkeit und einfachen Handhabung in der klinischen Routine meistens als ausreichend.

\section{Literatur}

${ }^{1}$ ASDA. Sleep-related breathing disorders in adults: Recommendations for syndrome definition and measurement techniques in clinical research. Sleep 1999; 22: 667-689

${ }^{2}$ Ayappa I, Norman RG, Krieger AC, Rosen A, O'Malley R, Rapoport DM. The use of a nasal cannula/pressure transducer system in detection of RERAs. Sleep 2000; Suppl 2, 23: A6

${ }^{3}$ Ballester E, Badia JR, Hernández L, Farré R, Navajas D, Montserrat JM. Nasal prongs in the detection of sleep-related disordered breathing in the sleep apnoea/hypopnoea syndrome. European Respiratory Journal, 1998; 11: 880-883

${ }^{4}$ Bloch KE, Li Y, Sackner MA, Russi EW. Breathing pattern during sleep disruptive snoring. Eur Respir 1997; 10: 576-586

${ }^{5}$ Exar EN, Collop NA. The Upper Airway Resistance Syndrome. Chest 1999; 115: 1127-1139

${ }^{6}$ Farré R, Montserrat JM, Rotger M, Ballester E, Navajas D. Accuracy of thermistors and thermocouples as flow-measuring devices for detecting hypopnoeas. Eur Respir J 1998; 11: 179182

${ }^{7}$ Gleeson K, Zwillich CW, Braier K, White DP. Breathing Route during Sleep. Am J Respir Crit Care Med 1986; 134: 115-120

${ }^{8}$ Hosselet JJ, Norman RG, Ayappa I, Rapoport DM. Detection of flow limitation with a nasal cannula/pressure transducer system. Am J Respir Crit Care Med, 1998; 157: 1461 - 1467

${ }^{9}$ Montserrat JM, Farré R, Ballester E, Felez MA, Pastó M, Navajas D. Evaluation of nasal prongs for estimating nasal flow. Am J Respir Crit Care Med 1997; 155: 211 - 215

${ }^{10}$ Rühle KH, Schlenker E, Randerath W. Upper airway resistance syndrome. Respiration, 1997; 64, Suppl. 1: 29-34

Prof. K.-H. Rühle

Klinik für Pneumologie

Klinik Ambrock

58091 Hagen 